\title{
Psychopharmacology and the Etiology of Psychopathologic States: Are We Looking in the Right Way?
}

Martin M. Katz, Ph.D. and James W. Maas, M.D.

\section{INTRODUCTION}

In a paper published in 1992, the present authors noted that in the past 3 decades research workers have produced remarkable advances in the pharmacologic treatment of major and minor psychopathologic disorders. These advances have had a major impact on our theories of the etiology of the mental disorders. However, our understanding of the neurobiologic and psychologic bases of these disorders has been confined mainly to the hypothesis-generating stage. We have not been able to establish definitively the nature of the neurobiologic basis of any known psychiatric disorder. Furthermore, although the discovery of the phenothiazines, lithium, and the tricyclic drugs in the 1950's launched an era of intensive research on therapeutic mechanisms of action, we are still not clear how any of these drugs act to bring about resolution of these disorders. Therefore, the question was raised in their earlier paper, "Have we been looking in the wrong places or in the wrong way?". No answer was suggested, only the observation and questions were raised.

From the Department of Psychiatry, Albert Einstein College of Medicine-Montefiore Medical Center (MMK), Bronx, New York; and the Department of Psychiatry, University of Texas Health Science Center at San Antonio (JWM), San Antonio, Texas.

Address correspondence to: Martin M. Katz, Ph.D., Department of Psychology, Montefiore Medical Center, 111 East 210th Street, Bronx, New York 10467.

Received April 26, 1993; revised October 19, 1993 and December 10, 1993; accepted December 27, 1993.

Presented at the Study Group: Neurobiology and Psychopathologic States: Are We Looking in the Right Place?, 1992 Meeting of the American College of Neuropsychopharmacology, San Juan.
Since that time, we have arranged a panel at a national meeting (ACNP 1992) to discuss this issue and have had a number of further discussions with colleagues. In this paper, we offer some thoughts as to where the field may have stumbled.

First, the problem may be that the neurobiology underlying psychopathologic states is too complex to be devined by our present research tools, even though some of them are very sophisticated: such as, MRI, PET, CAT scans, magnetoencephalography, echo planar imaging, GC-mass spectroscopy, and a variety of molecular biologic approaches. If this is the case, we will have to await the development of even more sophisticated methodologies. However, other possibilities for the causes of this problem have emerged from our discussions, and in contrast to the complexity issue, they lend themselves to change now using presently available methodologies. This suggestion and arguments in support of it are given in the remainder of this paper.

Borrowing from successes in other medical disciplines, in the field's biologic studies of mental disorders we have treated psychopathologic states as homogeneous diagnostic entities, such as depression and schizophrenia. This approach, since the introduction of more refined and reliable methods for diagnosis (namely, the Research Diagnostic Criteria [Spitzer et al. 1978]) has worked quite well in certain ways, such as evaluating the efficacy of a variety of drugs, delineating the typical time of onset of an illness, describing the natural history of an illness, and specifying gender distributions and demographics. However, in our opinion, the use of this model to elucidate the neurobiologic bases of these illnesses or the mechanisms of action of psychotropic drugs has led the field astray. In fact, psy- 
chopathologic states are not homogeneous entities but are composed of a variety of emotional, behavioral, and cognitive elements (herein referred to as components) that interact to create the disorder or are simply grafted onto the "core" of the disorder.

We suggest that each of these components may have its own neurobiologic basis (as might the core base of the disorder) and that when the illness is treated as a thing, the neurobiology that is related to the components may be confused with those related to the core. Similarly, hypotheses about the neurobiologic basis of the core disorder, such as the catecholamine hypothesis of the affective disorders, may actually be more relevant to a component of the depressive states: one of severe depression's major components is a very high level of anxiety. Some of the earlier identified antidepressant drugs have since been found to be potent anxiolytics as well (Kahn et al. 1985; Klein et al. 1978). Although they have demonstrated antidepressant properties, it may be that the well-known pharmacologic action of the drugs on brain amine systems is more related to their anxiolytic properties than to their effects on the disorder itself (Katz et al. 1987); that is, the antidepressant action of the drugs is due to some presently unknown biologic effect. If this very likely scenario is correct, then efforts to relate brain noradrenergic mechanisms in depression, per se, have a "built in" failure element.

Thus, much of what we have learned about depression over the past 4 decades, due to the introduction of these new drugs, is not being applied in our research. We continue, despite major advances in psychopharmacology, to adhere to a unitary model of disease.

Although this model in which psychopathologic state is a homogeneous entity has obvious advantages for clinical practice and for epidemiologic and phenomenologic research, it is not an effective device for investigating the multiple mechanisms of action of drugs. It is a global approach and, thus, has the effect of obscuring the process of behavioral change and ultimately limits our capacity to relate these drug-induced changes to etiology.

We suggest that the unitary model can actually work against further understanding of the underlying disorder and how the drug is working and that the alternative "componential" model serves more effectively in this type of research. The componential approach views the disorder in structural terms. The disorder consists of major components: emotional, behavioral, perceptual, cognitive, and somatic. The degree of the disturbance in each of these areas varies across patients and across phases of the disorder; change in any of these elements may be associated with the therapeutic action of a psychotropic drug. This does not mean that a given drug acts on the "core" etiology of the illness; only on one or more components. This model requires that a drug's actions on each of the major components be measured and that biochemical theories be examined as relating to the other components of the illness or to the interaction of these elements, not necessarily the core. Van Praag has presented a similar view (van Praag et al. 1975).

Additional examples might help to make our point. Direct clinical support for the linkage between schizophrenia and the brain dopamine system function is either negative or controversial (Bowers 1974; Rimon et al. 1971; Berger et al. 1980). However, we recently examined the relationship of cerebrospinal fluid (CSF) homovanillic acid (HVA) to a complex component of schizophrenia (psychosis) and found a strong correlation between the two $(n=24, r=0.70, p=.0001$ ) (Maas et al. unpublished observation). Several other groups have also found this strong correlation and have reported significant correlations between plasma HVA and severity of psychosis (Davis et al. 1991; Maas et al. 1988; Pickar et al. 1986).

Another illustrative example is that CSF 5-hydroxyindoleacetic acid (5-HIAA), a metabolite of serotonin (5-HT), was found to be low in a subgroup of depressed patients (Asberg et al. 1976a). A later report concluded that a low CSF 5-HIAA seemed to be associated with a proclivity of patients to commit suicide (Asberg et al. 1976b). This was thought generally to be congruent with a "5-HT theory of depression" and nonspecifically that a subtype of depression was associated with a dysfunction of brain 5-HT systems. Subsequent research with animals and humans however, has quite conclusively shown that brain 5 -HT systems regulate impulsivity and the expression of aggression (Linnoila et al. 1983; Soubrie 1986). Regardless of the experimental paradigm, this association between the 5-HT system and impulsive aggression has been replicated by most investigators. Clearly this has been a successful chapter in the history of psychobiology and biologic psychiatry, but consistent with the theme of this paper, impulsive aggression is not a diagnostic entity but rather a component of behavior and/or emotion that can be associated with pathologic or nonpathologic states.

From a multihospital study of depression, Bowden et al. (1985) reported that the biologic effects of tricyclic drugs on neurotransmitter systems were not specific to their therapeutic actions; namely, there were similar changes in treatment responders and nonresponders. In contrast, in severe depression, there were correlations of CSF concentrations of neurotransmitter metabolites with specific behavioral components when reexamined in the same hospitalized sample (Redmond et al. 1985). Cerebrospinal fluid 3-methoxy5-hydroxyphenylglycole was found to be significantly related to an "arousal" dimension of anxiety and its expressive facets, agitation, somatization, and sleep disorder but not a depressed mood-retardation dimension. 
This finding supported the hypothesis that the norepinephrine (NE) system is associated with the arousal and psychomotor aspects of the disorder, rather than with the core depression factor or with the disorder as a whole.

The major points in this paper can be illustrated in somewhat more detail by comparing certain results from an earlier study of the psychobiology of depression (Maas et al. 1980; Katz et al. 1979) with findings generated by other investigators who reasoned in this componential manner. In the earlier study, the structure of the disorder was reexamined in severely depressed hospitalized patients through the use of a range of behavioral, affective, and psychological performance measures. The investigation then focused on studying the nature and sequence of behavioral and emotional changes in patients who clearly responded to treatment with tricyclic drugs. This was done in a similar fashion to how the sequence of drug-induced neurochemical changes would be studied.

The work was based on earlier notions that tricyclic drugs have multiple behavioral effects on the clinical state (Kielholz, 1968) and that the drug actions on the 5-HT system result in different behavioral changes than actions on the NE system (Carlsson 1976).

\section{ON THE STRUCTURE OF DEPRESSION}

Examination in the previous study (Maas et al. 1980; Katz et al. 1979) of the average componential profile for a severely depressed sample of 104 patients indicated that the level of anxiety in those patients was almost as high as the level of depressed mood and that hostility was significantly higher than in healthy controls (Katz et al. 1984).

A principal components analysis* of the factor structure of the disorder identified three independent dimensions: two representing opposite states (in terms of arousal) coexisted in most severely depressed patients in this sample. They are a depressed, motorically retarded dimension, and an anxious, agitated, somatically aroused dimension. (The third dimension was hostility-interpersonal sensitivity). In this type of empirical analysis of sources of variance, these two dimensions

\footnotetext{
"The 11 "components" of the disorder are behavioral, affect, cognitive, and somatic constructs, such as, anxiety, motor retardation, and somatization. They were derived through factor analyses of behavioral rating, self-report, and psychological performance measures on a large sample of depressed patients. The constructs are correlated in various degrees with each other. The "dimensions" are uncorrelated or independent measures; they were derived through a principal components analysis (Morrison 1967) of the intercorrelations of the constructs. Each dimension is measured by combining two or more of the constructs with which it is most highly associated.
}

were found to have relatively equal influence in defining the clinical condition. Although the clinical concept of "anxious depression" is obviously not new, treating the two opposite psychomotor states as equal in presence has implications for how drug actions will be interpreted and for understanding the relative roles that the 5-HT and NE systems may play in the severe forms of the disorder.

\section{THE SEQUENCE AND NATURE OF TRICYCLIC DRUG ACTIONS IN DEPRESSION}

To exemplify how the componential approach was used to study the basis of drug action, the process of behavioral change in those patients who responded to the tricyclic drugs will be briefly described (Katz et al. 1987, 1991). To identify treatment responders stringent outcome criteria (not simply "marked" improvement) were set. Patients must have appeared to recover or nearly recover on a number of clinical indices to be classified as responders. The aim was then to identify the nature and sequence of changes in behavior in these patients over the 4 weeks of drug treatment.

The critical behaviors that appear to be first affected by the tricyclic drugs in the treatment of depression, and in which improvement is then followed by recovery in treatment-responsive patients are summarized in Figure 1. For amitriptyline, the behavioral changes that also correlated with drug concentrations in the

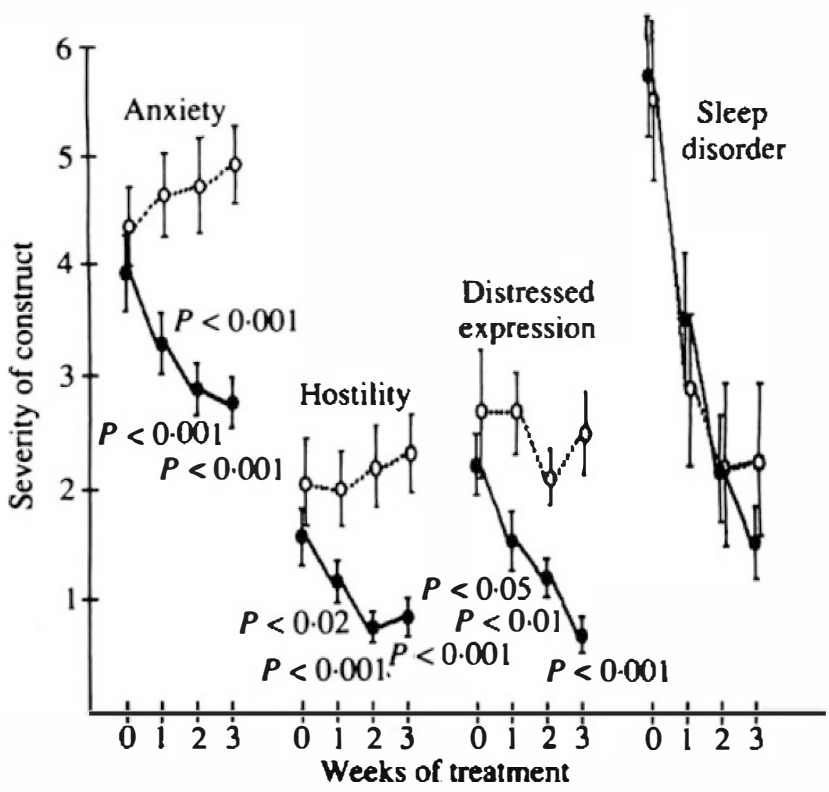

Figure 1. Differences between responder and nonresponder groups in behavioral changes during the first 21 days of amitriptyline treatment (0---0). Responders, $n=26,0 \ldots \ldots 0$, nonresponders, $n=12$. Adapted from Katz et al. (1991). 
plasma included: reductions in hostility, anxiety, and distressed (physical) expression. These were followed closely at $21 / 2$ weeks by an increase in social adaptation and reductions in depressed mood and motor retardation (Katz et al. 1991).

It was concluded from these behavioral changes that amitriptyline in responsive patients is associated with reducing hostility and anxiety, thus "calming" the organism (accounting in part for its efficacy in the anxiety disorders), and then with having a secondary "stimulant" action, resulting in a reduction in motor retardation (a similar pattern of action has since been described from this study for imipramine; our unpublished data).

The tricyclic drugs therefore appear to have multiple actions, both sedating and stimulating effects that appear to act on the two opposing affective qualities in the severe depressive disorder: that is, the depressedmotor retardation component and the anxiety-agitation or arousal component.

Having uncovered this dual action, one is then in a position, regarding the study of mechanisms of action, to determine which drug-induced changes in neurotransmitter functioning are associated with which changes in the behaviors and emotions. We will not carry the example further here but simply point to the latter step as a natural development for the componential approach. Please note that although the drug actions are related to components of the illness and much laboratory work suggests mechanisms by which central nervous system neurotransmitters are related to the components of emotion (for example, the now extensiveliterature on 5-HT and aggression), it does not mean that the systems involved in producing the "core" of the disorder have been adequately identified: the antidepressant drugs are possibly exerting their salutary effect by actions on an unknown (at present) mechanism.

\section{CONCLUSIONS}

As we indicated, the overreliance on diagnosis and the use of global measures of the disorder such as severity and improvement-common practices of clinical investigators in psychopharmacology - tends to obscure the actions of drugs and can mislead basic investigators in their research on mechanisms. Examples are the following:

1. Accepting the designation of tricyclics as "antidepressants" literally, as if the drugs are specific to the whole disorder of depression, has inhibited progress in understanding how they work. Without this designation, studies in the late 1970's that demonstrated that the drugs were equally effica- cious for phobias and generalized anxiety disorders (Klein et al. 1978; Kahn et al. 1979) would probably have surfaced much earlier. (Studies of the positive effects of imipramine on anxiety were in the literature in the early 1960 's.) In the work reported here, we learned that even when the study of tricyclic drug actions was confined to the depressive states, the effects on anxiety and hostility in treatment responders appeared earlier than those reported for depressive mood (Katz et al. 1991).

2. Despite the routine application of brief symptom scales in clinical drug trials and the use of scale items to measure symptoms such as the Hamilton Depression Scale (1960), the emphasis has been on overall change in the depressed state itself as the outcome measure, in other words, the severity of disorder or global improvement. The use of summary outcome measures in place of detailed examination of behavioral, affective, and cognitive functioning led to the conclusion that clinical changes were not effected in responsive patients until 2 or 3 weeks of treatment. This conclusion (based on inadequate evidence) contributed to obscuring the important early drug effects on certain behaviors and the emotions, aspects that were clearly changing by the end of the 1st week in treatment responders (Winokur 1980; Dimascio et al., 1979; Katz et al. 1991). These findings represented important information for basic investigators, who were led to believe that neurochemical effects that occurred later were more important than those that occurred earlier in the treatment process.

3. To apply basic findings regarding the associations of the 5-HT system with aggression or the NE system with arousal to the issues of the dynamics or causes of depression, it is necessary to measure the effects of the drugs on specific emotions and/or behaviors in the depressed state. Basic investigators in pharmacology and neurochemistry should be made aware of the importance or relevance of these emotions to the illness, to know for example, that anxiety and hostility play major roles in the clinical disorder of depression.

4. A basic message of this paper is also directed toward the manner in which new and emerging technologies such as molecular biology are applied to these research problems. The field is poised for a major advance in fostering understanding of molecular biologic processes on brain and on normal and abnormal behavior. Past history indicates, however, that wave after wave of new technologies have foundered in attempts to understand the biologic bases of mental illness. We suggest here that new approaches may be far more successful 
if they attempt to examine the relationships of these processes to the components of mental disorders prior to turning to the diagnostic model.

5 . The guiding principle is not to rely on general clinical practice and on a still "developing" diagnostic system to overly influence the design of clinical and basic research in psychopharmacology. We must try to introduce what we havelearned over the past few decades regarding the psychobiologic structure and components of these disorders into our ways of describing the syndrome and measuring how its components of behavior and emotion change. Only then will we be able to take full advantage of the remarkable conceptual and technical developments in neuropsychopharmacology.

In summary, we are suggesting that we have confused the multiple actions of psychotropic drugs on components of the main illness (anxiety and motor behavior in depression; psychosis in schizophrenia) with presumed effects on the "core" disturbance and, as such, have falsely hypothesized that certain central neurotransmitter systems are associated with the core of the illness rather than with its components only. We suggest that we still do not have any form and viable data that allows us to understand the main biologic defect(s) in any major psychopathologic state.

\section{ACKNOWLEDGMENT}

The work on this paper was supported in part by NIMH grants MH44403 and MH40936.

\section{REFERENCES}

Asberg M, Thoren P, Praskman L, Bertilsson L, Ringberger V (1976a): Serotonin depression: A biochemical subgroup within the affective disorders. Science 191:478-480

Asberg M, Praskman L, Thoren P (1976b): 5-HIAA in the cerebrospinal fluid. Arch Gen Psychiatry 33:1193-1197

Berger PA, Faull KF, KitkowskiJ, Anderson PA, Kraemer H, Davis K, Berchas JO(1980): CSF monoamine metabolites in depression and schizophrenia. Am J Psychiatry 137: 174-180

Bowden CL, Koslow SH, Hanin I, Maas JW, Davis JM, Robins $\mathrm{E}$ (1985): Effects of amitriptyline and imipramine on brain and amine neurotransmitter metabolites in cerebrospinal fluid. Clin Pharmacol Ther 37:316-324

Bowers MB (1974): Central dopamine tumover in schizophrenic syndromes. Arch Gen Psychiatry 81:50-54

Carlsson A (1976): The contribution of drug research to investigating the nature of endogenous depression. Pharmakopsychiatry 9:2-10

Davis KL, Davidson M, Mohs RC, Kendler KS, Davis BM,
Johns CA, DeNigris Y, Horvath TB (1991): Plasma homovanillic acid concentration and the severity of schizophrenic illness. Science 227:1601-1602

DiMascio A, Weissman MM, Prusoff BA, Neu C, Zwilling M, Klerman GL (1979): Differential symptom reduction by drugs and psychotherapy in acute depression. Arch Gen Psychiatry 36:1450-1456

Hamilton M (1960): A rating scale for depression. J Neurol Neurosurg Psychiatry 23:56-62

Kahn RJ, McNair DM, Lipman RS, Covi L, Rickels K, Downing R, Fisher S, Frankenthaler LM (1979): Imipramine and chlordiazepoxide in depression and anxiety disorders. II. Efficacy in anxious outpatients. Arch Gen Psychiatry 43:79-85

Katz MM, Koslow SH, Berman N, Secunda S, Maas JW, Casper R, Kocsis J, Stokes P (1984): Multivantaged approach to the measurement of behavioral and affect states for clinical and psychobiological research. Psychol Rep 55:619-791

Katz MM, Koslow SH, Maas J, Frazer A, Bowden CL, Casper R, Croughan J, Kocsis J, Redmond E (1987): The timing, specificity and clinical prediction of tricyclic drug effects in depression. Psychol Med 17:297-309

Katz MM, Koslow SH, Maas JW, Frazer A, Kocsis J, Secunda S, Bowden CL, Casper RC (1991): Identifying the specific clinical actions of amitriptyline: Interrelationships of behavior, affect and plasma levels in depression. Psychol Med 21:599-611

Katz MM, Secunda S, Hirschfeld RM, Koslow SH (1979): NIMH Clinical Research Branch Collaborative Program on the Psychobiology of Depression. Arch Gen Psychiatry 43:765-771

Kielholz P (1968): Die Behandlung endogener Depressionen mit Psychopharmaka. Deutsh Med Wschr 93:701

Klein DF, Zitrin CM, Woerner M (1978): Antidepressants, anxiety, panic and phobis. In Lipton MA, DiMascio A, Killam K (eds), Psychopharmacology: A Generation of Progress. New York, Raven Press, pp 1401-1410

Linnoila $\mathbf{M}$, Virkunnen $\mathbf{M}$, Scheimir $\mathbf{M}$, Nuutila $H$, Rimon R, Goodwin FK (1983): Low CSf 5-HIAA concentration differentiates impulsive from non-impulsive behavior. Life Sci 33:2609-2614

Maas JW, Contreras SA, Seleski E, Bowden CL (1988): Dopamine metabolism and disposition in schizophrenic patients: Studies using debrisoquen sulfate. Arch Gen Psychiatry 45:533-559

Maas JW, Katz MM (1992): Neurobiology and psychopathologic states: Are we looking in the right place? Biol Psychiatry $31: 757-758$

Maas JW, Katz MM, Frazer A, Stokes PE, Swann AC, Davis JM, Casper R, Berman N (1991): Current evidence regarding biological hypotheses of depression and accompanying pathophysiological processes: A critique and synthesis of results using clinical and basic research results. Integrat Psychiatry 7:155-161

Maas JW, Koslow SH, Davis J, Katz MM, Mendels J, Robins E, Stokes P, Bowden CL (1980): Biological Component of the NIMH Clinical Research Branch Collaborative Program in the Psychobiology of Depression I. Background and theoretical considerations. Psychol Med 10:759-776. 
Morrison DF (1967): Multivariate Statistical Methods. New York, McGraw-Hill

Pickar D, LaBarca R, Linnoila M, Roy A, Hommer D, Everett D, Paul SM (1986): Neuroleptic induced decrease in plasma homovanillic acid in schizophrenic patients. Arch Gen Psychiatry 43:669-676

RedmondDE, Katz MM, Maas JW, Swann A, Casper R (1986): Cerebrospinal fluid biogenic amine metabolite relationships with behavioral measurements in unipolar and bipolar depressed, manic and healthy control subjects. Arch Gen Psychiatry 43:938-947
Rimon R, Roos BE, Rakkolainen Y (1971): The content of 5-HIAA and HVA in the CSF of patients with acute schizophrenia. J Psychosom Res 15:375-378

Soubrie $P$ (1986): Reconciling the role of central serotonin neurons in human and animal behavior. Brain Sci 9:319-364

Van Praag HM, Korf J, Lakki JP, Schut WF (1975): Dopamine metabolism in depressions, psychoses, and Parkinson's Disease: The problem of the specificity of biological variables in behavior. Psychol Med 5:138-146

Winokur G (1980): What to do?, or what do we owe our residents? Biol Psychiatry 15:599-611 